\title{
RELAÇÃO UNIVERSIDADE-EMPRESA: PERFIL DOS CONTRATOS DE TECNOLOGIA E SUA DISTRIBUIÇÃO REGIONAL SEGUNDO DADOS DO FORMICT
}

\author{
UNIVERSITY-INDUSTRY RELATIONSHIP: TECHNOLOGY CONTRACTS PROFILING AND REGIONAL DISTRIBUTION \\ ACCORDING TO FORMICT DATA
}

Mayara Maria de Jesus Almeida ${ }^{1}$

Mauro Catharino Vieira da Luz ${ }^{2}$

Rogério Hermida Quintella ${ }^{3}$

${ }^{1}$ Doutora em Administração pela Universidade

Federal da Bahia.

Professora da Universidade do Estado da

Bahia.

mayarajalmeida@gmail.com

${ }^{2}$ Doutor em Engenharia de Produção pela POLI/USP.

Professor da Academia de Propriedade Intelectual do INPI. mauroluz@inpi.gov.br

\section{${ }^{3}$ Doutor em Gerenciamento Estratégico pela University of Brighton.}

Pró-Reitor de Pesquisa e Pós-Graduação da Universidade Federal do Sul da Bahia. rhquintella@gmail.com
Resumo: O artigo avalia e discute a transferência tecnológica a partir dos contratos declarados pelas Instituições Científicas e Tecnológicas (ICTs) brasileiras. Para tanto utilizou-se como fonte de dados o Formulário para Informações sobre a Política de Propriedade Intelectual das Instituições Científicas, Tecnológicas e de Inovação do Brasil (FORMICT) de 2017; indicadores do Diretório de Grupos de Pesquisa do CNPq, as bases patentárias do INPI; e da Pesquisa de Inovação Tecnológica (PINTEC)/IBGE. Os resultados apontam que pouco mais de $50 \%$ dos contratos declarados pelas ICTs no FORMICT se referem efetivamente à TT; entre eles, o número de contratos de licenciamento de direito de propriedade intelectual (DPI) foi o mais expressivo em quantidade, mas não em volume de recursos. Constatou-se também que aproximadamente $90 \%$ da negociação desses recursos está concentrada nas regiões Sul e Sudeste. Finalmente, que a relação entre os gastos e resultados com DPI mostra-se mais eficiente nas ICTs privadas.

Palavras-chave: Contratos. Transferência de Tecnologia. Instituições Científicas e Tecnológicas.

Abstract: The article evaluates and discusses technological transference from of the contracts signed by of the Brazilian Scientific and Technological Institutions (ICTS). For this purpose it was used as data source the FORMICT of 2017 and also was considered indicators available in Directory of Research Groups of CNPq and patent data-bases of INPI; and Brazilian Innovation Survey (PINTEC)/IBGE. The results indicate that a little more than $50 \%$ of the contracts declared by ICTs at FORMICT were really related to the TT. Among them, the number of agreement licenses of intellectual property rights (IPRs) was more expressive, though they are not the ones with the largest amount of negotiated resource. Besides, it was detected that approximately $90 \%$ of the negotiation of the resources is regionally concentrated in the South and Southeast. Finally, that the relationship between IPRs spending and results is more efficient in private ICTs.

Keywords: Contracts. Technology Transfer. Scientific and Technological Institutions. 


\section{Introdução}

O progresso tecnológico e a inovação são reconhecidos como motores do desenvolvimento econômico sobretudo em um contexto de maior intensidade de mudanças técnicas e concorrência em nível global. Com isso, muitos países têm investido em políticas de promoção e modernização tecnológica.

O progresso tecnológico nas organizações, em linhas gerais, pode ocorrer a partir de geração de conhecimento próprio (PD\&I) ou a partir da transferência tecnológica (TT). No primeiro caso, o conhecimento pode ser produzido internamente (modelo de inovação fechado), ou em parceria com fontes externas: outras organizações, instituições de pesquisa, clientes, fornecedores, etc. Já a TT pode referir-se à transposição de um conhecimento de base científica (tipicamente originado em $\mathrm{ICT}^{1}$ ), à produção (mercado); à migração de tecnologias entre diferentes campos de aplicação e, ainda, de localização geográfica (UNCTAD, 2014).

A inserção da $T T$ da universidade para a indústria se intensificou na década de $80 \mathrm{com}$ a publicação da Bayh-Dole Act nos Estados Unidos (Dias \& Porto, 20 14). Por se apresentarem como um importante locus de geração de novos conhecimentos de base científica e oportunidades tecnológicas, as universidades podem contribuir para inovação, tanto a partir do desenvolvimento de atividade de pesquisa e desenvolvimento (P\&D) com a empresa quanto com a transferência tecnológica a partir da cessão ou licenciamento de direitos de propriedade intelectual, do fornecimento de tecnologia.

Nesse contexto, o Brasil tem organizado políticas públicas de incentivo ao avanço tecnológico das empresas assim como a sua interação com ICTs. Desde os anos 2000, os governos têm implantado planos plurianuais com linhas de ação para a expansão e consolidação do sistema nacional à CT\&I por meio, principalmente, de apoio à formação de recursos humanos e à infraestrutura e fomento à pesquisa; à promoção da inovação na empresa através da subvenção econômica e o apoio à PD\&l em áreas estratégicas (MCTI, 2016). Dentre essas iniciativas, destaca-se a regulamentação da Lei 10.973 de $2004^{2}$ (Lei da Inovação), que, além de fornecer diretrizes legais (Dalmarco, Dewes, Zawislak \& Padula; 2011; Garnica \& Trokomian, 2009), instituiu a obrigatoriedade dos Núcleos de Inovação Tecnológica (NIT) nas ICTs para apoio às atividades de gestão e transferência da propriedade intelectual dessas instituições.

A fim de obter maior compreensão da temática, muitos estudos têm sido realizados nos últimos anos, principalmente a respeito da IUE (Lemos \& Cario, 2015; Noveli \& Segatto, 2012; Puffal, Trez \& Schaeffer, 2012), seus facilitadores (Carvalho, 2014), barreiras (Cunico, Cirani \& Teixeira, 2015) e sua relação com os elementos do sistema de inovação (Lemos \& Cario, 2015), Núcleos de Inovação Tecnológica, incubadoras (Adreassi \& Fugino, 2014) e parques tecnológicos (Novelli \& Segatto, 2012).

\footnotetext{
${ }^{1}$ Neste trabalho este termo está sendo utilizado para denominar instituições que realizem atividades de pesquisa básica ou aplicada de caráter científico ou tecnológico, não necessariamente órgão ou entidade da administração pública.

${ }^{2}$ Com alterações introduzidas pela Lei 13.243/2016, disponível em http://www.planalto.gov.br/ccivil_03/_ato2015-

2018/2016/lei/l13243.htm.
} 
Também, sobre aspectos relacionados aos seus resultados, como produção de propriedade intelectual (Closs, Ferreira, Sampaio \& Perin et al., 2012) e transferência de conhecimento e tecnologia (Sousa, Zambalde, Souki \& Veroneze, 2018; Viana, Jabour, Ramirez \& Cruz, 2018), seus canais, formas e intervenientes (Dalmarco et al., 2011; Dias \& Porto, 2014; Garnica \& Torkomian, 2009; Gomes, Gonçalo, Pereira \& Vargas, 2014; Soria \& Ferreira, 2012). A abordagem contratual, no entanto, ainda se encontra pouco explorada.

No sentido de contribuir com essa discussão, o presente artigo aborda o tema da transferência de tecnologia de ICTs no Brasil a partir dos contratos de tecnologia, nos quais são formalizados os direitos e deveres entre essas instituições e as empresas. Acredita-se que a análise desses instrumentos possa auxiliar no processo de avaliação das políticas de incentivo à CT\&I. Desse modo, o objetivo deste artigo é caracterizar os contratos de Transferência de Tecnologia (TT) realizados por ICTs no Brasil a partir das informações prestadas por estas no FORMICT, publicado em 2017. Para discussão desses dados, propõe-se aqui a verificação sobre como esses se relacionam com outras variáveis pertinentes à TT a partir de informações disponibilizadas pelo Diretório de Grupos de Pesquisa do CNPq, INPI e PINTEC.

Além desta introdução, este trabalho conta com a seção 2, que discute a importância da inovação para o desenvolvimento econômico e o papel que a cooperação entre capacidade técnicocientífica e indústria. Na seção 3, são apresentados os conceitos a respeito da transferência de tecnologia, a importância de celebração dos contratos de $T T$ e suas possíveis modalidades. Posteriormente, são descritos os procedimentos metodológicos da pesquisa na seção 4. Nas seções 5 e 6, os principais resultados encontrados são discutidos a luz da literatura. E, finalmente, na seção 7, são reunidas as considerações finais.

\section{Inovação e Interação Universidade-Empresa}

A informação e o conhecimento sempre tiveram importância para o desenvolvimento econômico, mas não com a intensidade atual. Embora a inovação tenha sido reconhecida como fator primordial para o desenvolvimento de empresas e da economia por Schumpeter nos anos 60, somente no final do século passado a temática passou a receber significativa atenção no âmbito de políticas públicas e da discussão acadêmica, principalmente após a reconfiguração de sua abordagem.

Tradicionalmente, o desenvolvimento da inovação era concebido como uma ordem sequencial de eventos que se inicia com a pesquisa científica básica, avança de maneira direta por níveis mais aplicados de pesquisa e termina com a sua aplicação e desenvolvimento, podendo gerar produtos de sucesso no mercado (Calderan \& Oliveira, 2013). Entretanto, a constatação de que numerosas e diversificadas melhorias podem ocorrer em todo o processo de inovação levou ao desenvolvimento de uma abordagem sistêmica que, segundo Mowery and Sampat (2010), está 
associada a uma relação mais interdisciplinar, pluralista "em rede" ou sistema de inovação (ver mais em Freeman, 1995, 1998; Lundvall, 2005; Nelson, 2002).

No contexto de crescente sofisticação das atividades de pesquisa e desenvolvimento necessárias à geração de inovações, a abordagem sistêmica de inovação mostra-se mais adequada e abre espaço para o desenvolvimento dos modelos de Triple-Helix (Etzkowitz \& Leydesdorrff, 2000) e Open Innovation (Chesbrough, 2003). Nesses, o papel das universidades e instituições de pesquisa adquire relevância uma vez que se apresentam como loci principais de geração de novos conhecimentos (Etzkowitz \& Zhou, 2017; Perkmann et al., 2013).

De acordo com Ankrah and Tabaa (2015), a literatura sobre o tema IUE tem crescido substancialmente e abordado as motivações que levam ao estabelecimento da parceria, as possibilidades de formatos organizacionais, o processo de cooperação em si, suas barreiras e facilitadores e, ainda, os resultados esperados pelas partes. Esses, de acordo com Perkmann Neely \& Walsh (2011), variam em função do tipo de interação estabelecida, enquanto os de maior relevância geralmente estão associados aos vínculos com alto envolvimento relacional.

A apropriação do conhecimento científico pelo setor produtivo, no entanto, não é um processo trivial, pois necessita de conhecimento e experiência na operacionalização dos procedimentos relacionados à proteção jurídica do conhecimento (sigilo, confidencialidade, propriedade intelectual) e transferência de tecnologia (negociação, celebração de contratos e capacidade de absorção).

\section{Transferência Tecnológica e contratos}

Não há um consenso a respeito da definição do processo de transferência tecnológica, sua conceituação depende do contexto de ocorrência. De acordo com a United Nations Conference on Trade and Development - UNCTAD (2014), a transferência tecnológica pode referir-se à transposição de um conhecimento de base científica (instituição científica tecnológica) à produção (mercado); migração de tecnologias entre diferentes campos de aplicação e, ainda, de localização geográfica (UNCTAD, 2014).

A transferência de tecnologia abordada nesse estudo refere-se especificamente à transposição de tecnologia desenvolvida por ICT para o setor produtivo. Como pontuam Amesse and Cohendet (2001), no contexto da economia do conhecimento, o processo de TT envolve os caminhos que as empresas e outras instituições utilizam para gerir o conhecimento, em particular, a co-evolução de suas capacidades de absorção e suas estratégias de transmissão de conhecimento. Isso porque, diferentemente do que ocorre com a aquisição de um bem físico, que pressupõe a sua transposição de um local para outro, a transferência de tecnologia pressupõe um conhecimento necessário à utilização do ativo adquirido. 
Nesse sentido, a eficácia do processo de TT depende da capacidade de absorção (apoiada nas competências de desenvolvimento, adaptação e aprendizagem), competência para operacionalizar os procedimentos jurídicos de proteção e gestão do processo, no qual o contrato tem papel fundamental.

Para Luz (2015), os contratos de tecnologia são acordos formais realizados entre organizações para estabelecimento de termos e condições para transação de direitos relacionados a informações. Ainda, segundo o autor, esses contratos podem ser utilizados nas diversas modalidades de transações, na regulamentação de acordos de colaboração para desenvolvimento de tecnologias, também para operações de compra e venda de ativos intangíveis, como o licenciamento/cessão de direitos de propriedade intelectual.

Os direitos de propriedade intelectual (DPIs) envolvem um conjunto de regras, procedimentos e instituições que regulamentam a apropriabilidade, a transferência, o acesso e o direito ao uso do conhecimento e de ativos intangíveis (Zucoloto, 2013). Barbosa (2003) esclarece que sua expressão, antes restrita aos direitos autorais, é ampliada após a Convenção da OMPI, passando a designar a

soma dos direitos relativos às obras literárias, artísticas e científicas, às interpretações dos artistas intérpretes e às execuções dos artistas executantes, aos fonogramas e às emissões de radiodifusão, às invenções em todos os domínios da atividade humana, às descobertas científicas, aos desenhos e modelos industriais, às marcas industriais, comerciais e de serviço, bem como às firmas comerciais e denominações comerciais, à proteção contra a concorrência desleal e todos os outros direitos inerentes à atividade intelectual nos domínios industrial, científico, literário e artístico (Barbosa, 2003, p. 10).

O estímulo à estruturação e harmonização desses instrumentos está relacionado a melhores condições de atrair investimentos estrangeiros, ao garantir que as inovações não seriam copiadas por concorrentes locais, e facilitação de governança de transações tecnológicas em escala global (UNCTAD, 2014; Zucoloto, 2013). Conforme pontuam Garnica \& Torkomian (2009), uma vez que a missão da universidade não contempla a produção de bens e serviços, o seu interesse na proteção do conhecimento deve ter como finalidade torná-lo viável economicamente a partir de sua transferência para o setor produtivo que possa implementá-lo.

No contexto das IUE, então, a proteção dos direitos de propriedade intelectual pela ICT facilita a transferência do ativo intangível, resultado de atividade científica, para que a empresa realize o desenvolvimento tecnológico criando novos produtos, processos, serviços ou aprimorando-os, porque delimita mais claramente o objeto a ser transferido e previne a utilização não autorizada. (UNCTAD, 2014).

Estudos recentes (Sousa et al., 2018; Viana et al., 2018) têm apontado que, embora tenha ocorrido um aumento de pedidos de Pl pelas universidades nos últimos anos, a transferência de tecnologia não cresceu com a mesma intensidade. Para Sousa et al. (2018), esse quadro está associado à falta de postura empreendedora e à limitada utilização das estratégias de comunicação 
pelas universidades, uma vez que seus resultados científicos são muitas vezes distantes da aplicação comercial. Contribuem ainda para esse cenário a ausência de legislação clara e específica de estímulo à TT, baixa autonomia, infraestrutura adequada e pessoal com capacidade técnica nos NITs para estudos de viabilidade econômica e técnica das tecnologias desenvolvidas (Viana et al., 2018).

O aumento do número de DPI então, não necessariamente implica o desenvolvimento de inovação, pois, para que ocorra a TT, é necessário que o ofertante tenha o interesse e disponibilidade para transferir o ativo intangível e o demandante, condições de absorvê-lo, independentemente de estar protegido por direito de propriedade, como apontado anteriormente (Zucoloto, 2013). Neste sentido, a formalização dos termos de acordo a partir do estabelecimento do contrato de tecnologia é fundamental para reduzir os riscos inerentes a essa transação (Luz, 2015; UNCTAD, 2014).

Não há legislação única para a regulamentação dos contratos de propriedade industrial e de transferência de tecnologia no Brasil. De acordo com Barbosa (2003), estes são regulados por um conjunto disperso e desconexo de normas. Estas incluem legislação tributária; legislação de direito da concorrência; regulamentação da aplicação do capital estrangeiro e remessas de valores; Lei da Propriedade Industrial; efeitos internos do tratado OMC/TRIPS e, ainda, dispositivos do código do consumidor.

Ainda, segundo o autor, vários são os objetos do comércio de propriedade industrial e tecnologia, os quais podem ser divididos em quatro tipos: (i) contratos de PI, que podem versar sobre licença e cessão de direitos de propriedade industrial, como patentes, marcas, desenhos industriais e softwares; (ii) contratos de fornecimento de tecnologia, que não envolvem direito de propriedade industrial formalmente constituídos, como know how e segredos industriais; (iii) contratos de projeto, que consistem em produto imaterial da aplicação de uma tecnologia. Diferenciam-se do fornecimento de tecnologia pelo fato do conhecimento transacionado não estar diretamente vinculada ao objeto da empresa demandante e ser incorporada por ela, como, por exemplo, projetos de engenharia; (iv) contratos de serviços técnicos, que envolvem a transação de objetos, técnicas, métodos de aplicação de tecnologia que não se configuram como direitos de PI e nem mesmo chegam a criar um produto imaterial, mas estão submetidos às regras de mercado de tecnologia, como treinamento, por exemplo.

A Lei de Propriedade Industrial (n. 9.279, 1996) estabelece que, para produzirem efeitos em relação a terceiros, os contratos de tecnologia devem ser registrados pelo Instituto Nacional de Propriedade Industrial (INPI). Conforme publicado em sua página na internet ${ }^{3}$, as relações contratuais de transferência de tecnologia averbadas pelo INPI podem ocorrer em três modalidades. As características do que cada uma dessas visa autorizar estão resumidas no Quadro 1. Relacionando-as com a classificação proposta por Barbosa (2003), apresentada anteriormente, o contrato de propriedade industrial se restringe apenas à transação de patentes, desenho industrial e marcas; os

${ }^{3}$ Disponível em http://www.inpi.gov.br/menu-servicos/transferencia/transferencia-de-tecnologia-mais-informacoes 
contratos de fornecimento de tecnologia, de projeto e serviços técnicos estão englobados em aquisição de conhecimentos tecnológicos e é acrescentada a modalidade franquia.

Quadro 1 - Modalidades de contratos averbados no INPI

Tipo de contrato

Cessão e licença de

direitos de propriedade industrial

conhecimentos

tecnológicos

\section{Objetivo}

exploração por terceiros do objeto de patentes ou desenho industrial, regularmente depositados ou concedidos no país. uso de marca, regularmente depositada ou registrada no país. fornecimento de tecnologia, que estipula as condições para a aquisição de conhecimentos e de técnicas não amparados por direito de propriedade intelectual no Brasil (Know how).

serviços de assistência técnica e científica, que incluem a obtenção de técnicas para elaborar projetos ou estudos e a prestação de alguns serviços especializados.

Franquia serviços, transferência de tecnologia e transmissão de padrões, além de uso de marca ou patente.

Fonte: Elaborado pelos autores a partir de dados disponíveis no INPI, 2014.

O estabelecimento de contratos de compra e venda de tecnologia entre universidades e empresas constitui uma etapa avançada no processo de desenvolvimento tecnológico, uma vez que é o indicador mais próximo da efetiva ocorrência da transferência da tecnologia desenvolvida na universidade para aplicação industrial pela empresa, podendo gerar resultados econômicos para universidade a partir do recebimento de recursos financeiros pelo fornecimento de serviços técnicos, de tecnologia e, ainda, pelo licenciamento ou cessão dos DPI. O recebimento dessas receitas é legalmente permitido às ICTs públicas, conforme a Lei de Inovação Tecnológica, no 10.973/2004.

\section{Procedimentos metodológicos}

Para a caracterização dos contratos de Transferência de Tecnologia (TT) celebrados por ICTs no Brasil, foi realizada uma pesquisa descritiva de abordagem qualitativa por meio da coleta de dados secundários no relatório do Formulário para Informações sobre a Política de Propriedade Intelectual das Instituições Científicas e Tecnológicas do Brasil (FORMICT).

O preenchimento desse formulário é obrigatório às ICTs públicas e privadas beneficiadas pelo poder público. Conforme o Artigo 17 da Lei de Inovação no 10.973/2004, essas instituições devem prestar informações anuais sobre sua política de propriedade intelectual, criações desenvolvidas, proteções requeridas e concedidas e contratos de licenciamento ou transferência de tecnologia firmados ao MCTIC. Para tanto, desde 2005, esse ministério tem disponibilizado um formulário eletrônico para que as ICTs prestem as informações, a partir do qual também se elabora um relatório oficial anual para apresentação dos dados consolidados (denominado FORMICT, desde 2006), incluindo informações relativas aos contratos de transferência de tecnologia.

O formulário fica disponível para preenchimento entre os meses de fevereiro e março de cada ano para que as ICTs insiram as informações referentes às atividades realizadas no ano anterior (MCTIC, 2017). Dessa maneira, os dados analisados compreendem aqueles apresentados no relatório 
do FORMICT de 2017, referente ao ano-base 2016, última edição publicada no período de coleta dos dados para a realização da pesquisa. Os relatórios dos anos anteriores não foram considerados nesta pesquisa devido à não padronização das informações divulgadas e, principalmente, por estas não se constituírem resultados de uma mesma amostra, uma vez que o número de instituições respondentes do FORMICT tem crescido ao longo do tempo.

Para uma análise mais abrangente dos dados obtidos no FORMICT, foi realizada uma pesquisa sobre outros indicadores pertinentes ao tema. Ou seja, buscou-se informações a respeito dos tipos de interação U-E registrados pelos coordenadores de grupo de pesquisa cadastrados no CNPq; estatísticas sobre depósitos de patentes do INPI; investimentos em P\&D e atividades inovativas realizadas pelas empresas brasileiras nos respectivos sítios da Internet.

Considera-se que a análise conjunta desses dados seja relevante por se tratar de indicadores de entrada dos contratos de tecnologia celebrados pelas ICTs. Nesse sentido, o comportamento desses indicadores foi avaliado em 2014, momento anterior ao período de preenchimento do FORMICT pelas ICTs porque é possível que os contratos celebrados em 2016 guardem alguma relação com as atividades desenvolvidas anteriormente. Também foram registrados quais os índices apresentados por esses indicadores dez anos antes, em 2004, a fim de verificar o comportamento apresentado por eles após a regulamentação da Lei de inovação e demais políticas de incentivo à C,T\&I.

Os resultados são apresentados com apoio de tabelas e gráficos e compreendem, além do perfil das ICTs que compuseram a população de respondentes do FORMICT, os seus indicadores relativos à celebração de contratos de tecnologia e indicadores de atividades relacionadas à TT a partir dos dados obtidos no Diretório de grupos de Pesquisa do CNPq e INPI.

\section{Tipologia dos contratos de tecnologia das ICTs brasileiras}

De acordo com o relatório FORMICT, 278 instituições preencheram o formulário eletrônico em 2016. O perfil das ICTs respondentes é bastante diversificado (conforme Tabela 1), abrangendo, além das 193 instituições públicas (69,4\%), também 85 instituições privadas (30,6\%), que só têm obrigatoriedade de preenchimento do formulário se, de alguma forma, beneficiadas pelo poder público (MCTIC, 2017).

Com relação ao perfil das instituições que declararam ter celebrado contratos de tecnologia, 42 são de natureza pública (72\%) e 16 instituições privadas (28\%), distribuídas conforme Tabela 1. Esse resultado confirma a importância das instituições públicas de pesquisa no país, sobretudo as universidades e institutos de pesquisa como locus principal de geração de conhecimento técnicocientífico, o que coaduna com a abordagem sistêmica de inovação. 
Tabela 1 - Perfil das ICTs que declararam possuir contrato de tecnologia em 2017

\begin{tabular}{|c|c|c|c|c|}
\hline \multirow[t]{2}{*}{ Perfil } & \multicolumn{2}{|c|}{$\begin{array}{c}\text { ICTs } \\
\text { respondentes do } \\
\text { FORMICT }\end{array}$} & \multicolumn{2}{|c|}{$\begin{array}{l}\text { ICTs que declaram ter } \\
\text { celebrado contratos de } \\
\text { tecnologia }\end{array}$} \\
\hline & Qte & $\%$ & Qte & $\%$ \\
\hline Instituições de Ensino Superior & 135 & 48,6 & 29 & 50,0 \\
\hline Institutos de Pesquisa & 68 & 24,5 & 17 & 17,0 \\
\hline Institutos de Educação Profissional e Tecnológica & 40 & 14,4 & 5 & 8,6 \\
\hline Outros possíveis & 22 & 7,9 & 7 & 12,1 \\
\hline TOTAL & 278 & 100,0 & 58 & 100,0 \\
\hline
\end{tabular}

Fonte: Elaborada pelos autores a partir do FORMICT, 2017.

Dentre as modalidades de contratos apresentadas por Barbosa (2003), duas estão presentes no FORMICT: contrato de propriedade intelectual, representado na Tabela 2 pelas linhas 1 e 7; contratos de fornecimento de tecnologia e serviços técnicos associados que não envolvem direito de PI formalmente constituído, representados nas linhas 3 e 10. Desse modo, observa-se que, dentre os 1957 contratos de tecnologia declarados pelas ICTs no FORMICT, existem modalidades que não se referem efetivamente à transferência tecnológica, mas a atividades anteriores ao seu processo. Excluindo-se o número de contratos referentes a essas atividades, restaram 1029 contratos de transferência de tecnologia (linhas sombreadas na Tabela 2). Esses representam 52,6\% do total de contratos realizados. 
Tabela 2 - Distribuição dos contratos de tecnologia celebrados pelas ICTs por objeto em 2017

\begin{tabular}{|c|c|c|c|}
\hline Objeto & $\begin{array}{l}\text { Qde. de ICTs que } \\
\text { declararam ter } \\
\text { celebrado contrato }\end{array}$ & $\begin{array}{l}\text { Qtde de } \\
\text { contratos }\end{array}$ & $\%$ \\
\hline $\begin{array}{l}\text { Contrato de licenciamento de direitos de propriedade } \\
\text { intelectual (patente; desenho industrial; marca; programa de } \\
\text { computador; topografia de circuito integrado; cultivar; obra } \\
\text { literária, artística ou científica; outros). }\end{array}$ & 30 & 823 & 42,1 \\
\hline $\begin{array}{l}\text { Acordo de parceria de pesquisa, desenvolvimento e inovação } \\
\text { (atividades conjuntas de pesquisa científica, e/ou tecnológica e } \\
\text { desenvolvimento de tecnologia, produto ou processo). }\end{array}$ & 34 & 485 & 24,8 \\
\hline $\begin{array}{l}\text { Contrato de know how (envolvendo ativos intangíveis não } \\
\text { amparados por direito de PI), assistência técnica (contratação } \\
\text { de soluções técnicas ou capacitação ou treinamento) e demais } \\
\text { serviços. }\end{array}$ & 15 & 159 & 8,1 \\
\hline Acordo de confidencialidade. & 12 & 133 & 6,8 \\
\hline Contrato de co-titularidade. & 13 & 84 & 4,3 \\
\hline Acordo de transferência de material biológico. & 6 & 19 & 1,0 \\
\hline $\begin{array}{l}\text { Contrato de cessão de direitos de propriedade intelectual } \\
\text { (patente; desenho industrial; marca; programa de } \\
\text { computador; topografia de circuito integrado; cultivar; obra } \\
\text { literária, artística ou científica; outros). }\end{array}$ & 2 & 2 & 0,1 \\
\hline $\begin{array}{l}\text { Contrato ou convênio de permissão de utilização de } \\
\text { laboratórios, equipamento, instrumentos, materiais e } \\
\text { instalações por empresas nacionais e organizações de direito } \\
\text { privado sem fins lucrativos e voltados para atividades de } \\
\text { pesquisa. }\end{array}$ & 4 & 76 & 3,9 \\
\hline $\begin{array}{l}\text { Contrato ou convênio de compartilhamento de laboratórios, } \\
\text { equipamento, instrumentos, materiais e instalações com } \\
\text { microempresas e empresas de pequeno porte em atividades } \\
\text { voltadas à inovação tecnológica, para consecução de } \\
\text { atividades de incubação. }\end{array}$ & 5 & 27 & 1,4 \\
\hline $\begin{array}{l}\text { Contrato ou convênio de uso do capital intelectual em projetos } \\
\text { de pesquisa, desenvolvimento e inovação. }\end{array}$ & 5 & 45 & 2,3 \\
\hline Outros & 13 & 104 & 5,3 \\
\hline TOTAL & 139 & 1957 & 100,0 \\
\hline
\end{tabular}

Fonte: FORMICT, 2017

Os tipos de contratos mais celebrados pelas ICTs foram os de licenciamento de direitos de propriedade intelectual (42,1\%), seguido de acordo de parceria $(24,8 \%)$ e dos contratos de know how (8,1\%), conforme Tabela 2. Esses números sugerem um amadurecimento das ICTs no que se refere à proteção de sua propriedade intelectual, resultado das políticas de incentivo à CT\&I implantas no Brasil a partir dos anos 2000, conforme mencionadas anteriormente. De modo geral, observa-se que o estabelecimento de infraestrutura de auxílio a proteção das tecnologias desenvolvidas pelas universidades e de regulamentações internas sobre a legalidade de sua exploração tendem a favorecer o aumento da ocorrência da TT envolvendo o uso de PI (Garnica \& Torkomian, 2009). 
Dados históricos referentes à atividade de patenteamento no Brasil revelam que, apesar da relativa estabilidade no número de depósito por residentes no INPI ${ }^{4}$ desde a regulamentação da primeira Lei de Inovação Tecnológica, houve significativo incremento da participação das ICTs nesse quantitativo. Em 2005, o INPI registrou 7.346 depósitos por residentes, dos quais 296 foram realizados por universidades e institutos de pesquisa; já em 2015, esses números foram de 7.344 e 534, respectivamente (INPI, 2017). Ou seja, em dez anos, a participação das ICTs passou de 4,0 para 7,3\% do total de depósitos de patentes por residentes no INPI.

O incremento das atividades de patenteamento e comercialização de tecnologia havia sido detectado nas universidades paulistas 5 por Garnica e Torkomian (2009). Viana et al. (2018) também constataram o crescimento nos pedidos de patentes pelo setor acadêmico a partir de análise documental em fontes de dados secundárias sobre as atividades de PI e TT das universidades brasileiras. Entretanto, ainda de acordo com os autores, a TT entre universidade e indústria no Brasil encontra-se em estágio embrionário. Como apontam Dalmarco et al. (2011), se a PI não é efetivamente transferida para o setor produtivo para que a nova tecnologia seja convertida em produtos e serviços, a proteção é inútil. Para alteração desse quadro, Sousa et al. (2018) sugerem que as universidades desenvolvam capacidades de marketing e confiram maior atenção às necessidades do mercado e da sociedade, para tanto urge maior e melhor integração entre esses atores, ou seja, a IUE.

De fato, após a regulamentação da Lei de Inovação também houve um incremento do número de grupos de pesquisa vinculados às universidades, cadastrados no $\mathrm{CNPq}^{6}$, que declararam interagir com o setor produtivo. O mesmo foi observado em relação ao número de relacionamentos estabelecidos. A partir de consultas realizadas na base dados do CNPq, verificou-se que, no censo de 2004, 2.151 grupos relataram algum relacionamento com empresa. Em 2014 esse número foi de 9.348, o que representa um incremento de aproximadamente 340\%. O número de relacionamentos, por sua vez, apresentou crescimento de aproximadamente 5 vezes: em 2004 foram declaradas 5.730 interações distribuídas entre os 14 tipos de relacionamentos; em 2014, esse quantitativo foi de 28.246, conforme Tabela 3.

\footnotetext{
${ }^{4}$ Identificação da natureza jurídica do 10 depositante residente.

5 De acordo com Dalmarco et al., (2011), essas universidades representam 79,5\% das patentes depositadas pelas universidades brasileiras entre 1979 e 2007.

${ }^{6}$ Apesar de esse dado não abranger cooperação das empresas com instituições de pesquisa, acredita-se que seja um importante indicador a jusante do processo TT na análise dos contratos de tecnologia pesquisados.
} 
Tabela 3 - Número de relacionamentos declarados pelos grupos de pesquisa do CNPq por tipo de relacionamento

\begin{tabular}{|c|c|c|c|}
\hline \multirow{2}{*}{ Tipo de relacionamento } & \multicolumn{3}{|c|}{ Quantidade declarada pelos grupos 7} \\
\hline & 2004 & 2014 & Crescimento (\%) \\
\hline $\begin{array}{l}\text { Desenvolvimento de software não-rotineiro para o grupo pelo } \\
\text { parceiro }\end{array}$ & 110 & 231 & $210 \%$ \\
\hline $\begin{array}{l}\text { Atividades de engenharia não-rotineira, inclusive o } \\
\text { desenvolvimento/fabricação de equipamentos para o grupo }\end{array}$ & 75 & 284 & $379 \%$ \\
\hline $\begin{array}{l}\text { Fornecimento, pelo parceiro, de insumos materiais para as } \\
\text { atividades de pesquisa do grupo sem vinculação a um projeto } \\
\text { específico de interesse mútuo }\end{array}$ & 368 & 323 & $88 \%$ \\
\hline $\begin{array}{l}\text { Atividades de engenharia não-rotineira, inclusive o } \\
\text { desenvolvimento de protótipo, cabeça de série ou planta- } \\
\text { piloto para o parceiro }\end{array}$ & 239 & 388 & $162 \%$ \\
\hline Desenvolvimento de software para o parceiro pelo grupo & 208 & 406 & $195 \%$ \\
\hline $\begin{array}{l}\text { Treinamento de pessoal do grupo pelo parceiro, incluindo } \\
\text { cursos e treinamento "em serviço" }\end{array}$ & 177 & 1075 & $607 \%$ \\
\hline $\begin{array}{l}\text { Transferência de tecnologia desenvolvida pelo parceiro para o } \\
\text { grupo }\end{array}$ & 207 & 1417 & $685 \%$ \\
\hline $\begin{array}{l}\text { Fornecimento, pelo grupo, de insumos materiais para as } \\
\text { atividades do parceiro sem vinculação a um projeto específico } \\
\text { de interesse mútuo }\end{array}$ & 53 & 1456 & $2747 \%$ \\
\hline $\begin{array}{l}\text { Atividades de consultoria técnica não englobadas em } \\
\text { qualquer das categorias anteriores }\end{array}$ & 437 & 1678 & $384 \%$ \\
\hline $\begin{array}{l}\text { Treinamento de pessoal do parceiro pelo grupo, incluindo } \\
\text { cursos e treinamento "em serviço" }\end{array}$ & 389 & 1761 & $453 \%$ \\
\hline $\begin{array}{l}\text { Transferência de tecnologia desenvolvida pelo grupo para o } \\
\text { parceiro }\end{array}$ & 835 & 1900 & $228 \%$ \\
\hline $\begin{array}{l}\text { Outros tipos predominantes de relacionamento que não se } \\
\text { enquadrem em nenhum dos anteriores. }\end{array}$ & 316 & 3123 & $988 \%$ \\
\hline $\begin{array}{l}\text { Pesquisa científica com considerações de uso imediato dos } \\
\text { resultados }\end{array}$ & 1.447 & 6.715 & $464 \%$ \\
\hline $\begin{array}{l}\text { Pesquisa científica sem considerações de uso imediato dos } \\
\text { resultados }\end{array}$ & 869 & 7489 & $862 \%$ \\
\hline Total & 5730 & 28246 & $493 \%$ \\
\hline
\end{tabular}

Fonte: Elaborada pelos autores a partir do DG CNPq, 2017.

Embora não seja possível estabelecer uma relação direta entre o perfil dos relacionamentos que os grupos de pesquisa declararam manter com empresa no diretório de grupos de pesquisa do CNPq e os tipos contratos de transferência de tecnologia declarados pelas ICTs ao FORMICT, o crescimento desses dados, no período analisado, mostra-se coerente nas duas fontes e sinaliza avanços no sentido de maior aproximação da comunidade científica e setor produtivo. Afinal, além de ser realizada por um maior número de pesquisadores, a interação passou a ocorrer com mais frequência em diferentes modalidades e tem sido formalizada pelas instituições. Este cenário sugere significativa expansão em direção à consolidação do sistema nacional de inovação, importante linha de ação fomentada pela política de CTI no país nos últimos anos.

\footnotetext{
${ }^{7} \mathrm{O}$ número dos tipos de relacionamento é diferente do quantitativo de grupos de pesquisa porque "foi permitido ao grupo informar até três tipos de relacionamento para cada empresa mencionada" (CNPq, 2017).
} 
Conforme FORMICT, as instituições declararam ter recebido $\mathrm{R} \$ 437,8$ milhões oriundos dos 1957 contratos de tecnologia. Os dados presentes no relatório permitem ainda identificar o montante de recursos por tipo de contrato celebrado, conforme Figura 1. A partir da comparação desses dados com aqueles apresentados na Tabela 2, é possível observar que o montante de recursos oriundos dos diferentes objetos de contrato não é proporcional à quantidade de contratos.

Figura 1 - Montante de recursos por objeto dos contratos de tecnologia

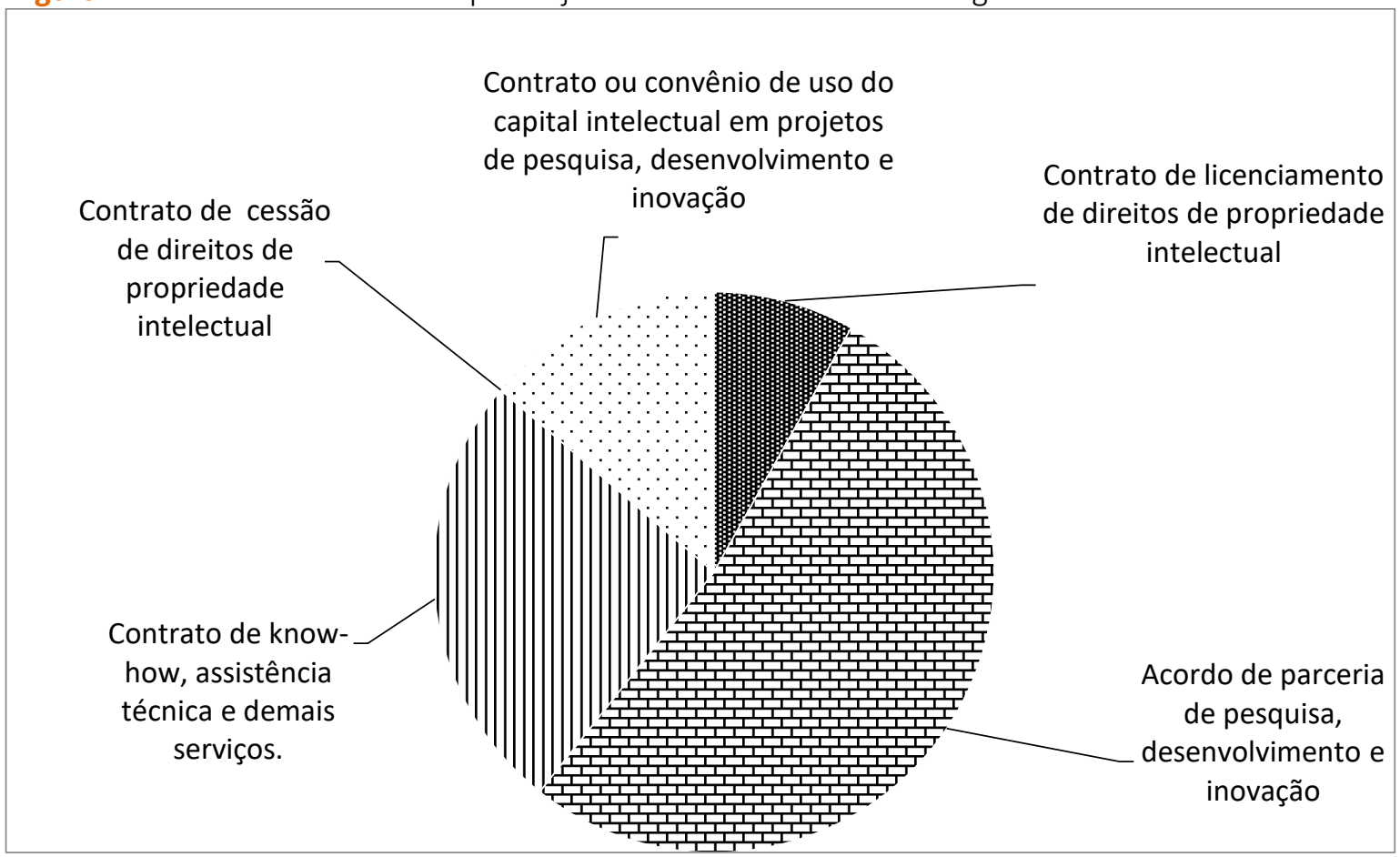

Fonte: Elaborada pelos autores a partir do FORMICT, 2017.

A modalidade contratual com maior volume de recursos foi o acordo de parceria de pesquisa, desenvolvimento e inovação ${ }^{8}$, com $\mathrm{R} \$ 221,7$ milhões. Na sequência, os contratos de know how apresentam o segundo maior montante, $\mathrm{R} \$ 108$ milhões; enquanto que a modalidade de contrato mais representativa em termos de quantidade apresentou um montante de $R \$ 34,4$ milhões, Figura 1.

Acredita-se que esses fatos sejam explicados pelo período de pagamento da operação da transferência de tecnologia. Para a realização da pesquisa, desenvolvimento e inovação, é necessária a disponibilização dos recursos no início do processo, inclusive para compra de equipamentos, materiais. Desse modo, é possível que a "entrada" de recursos nesta operação seja em um curto período de tempo posterior à celebração do contrato para o desenvolvimento da pesquisa ou prestação de serviço. Enquanto que o pagamento de licenciamento de DPI (ou pelo menos parte dele) não ocorre no ato da celebração do acordo, mas, sim, em um médio período de tempo, geralmente após o produto e serviço já estarem disponibilizados no mercado.

\footnotetext{
${ }^{8}$ Apesar de este processo não ser considerado como transferência tecnológica, foi mantido, na apresentação de dados na Figura 1 e Tabela 3 , dada a sua significativa contribuição no quantitativo de recursos obtidos com os contratos de tecnologia declarados pelas ICTs no FORMICT 2017
} 
Os formulários da base de dados do Diretório de Grupos de Pesquisa do CNPq permitem que os grupos declarem o tipo de remuneração envolvida nos relacionamentos que os mesmos estabelecem com empresas. Embora não seja possível associá-la a um tipo específico de contrato de transferência de tecnologia, um comparativo entre os dados de 2004 e 2014 revelou um crescimento da frequência de transferência de recursos entre grupos e empresas. Em 2004, 4.552 grupos declararam que suas relações envolviam transferência de recursos com empresas e, desses, 1070 declararam ter recebido recursos financeiros. Em 2014, esses números foram de 26.493 e 2690, respectivamente. O que sinaliza que a remuneração tem acompanhado o aumento do número de interações observadas no período.

Percebeu-se na análise do FORMICT a distribuição do montante de recursos negociados nos contratos de tecnologia por região apresentado na Tabela 4. As ICTs das regiões Sul e Sudeste somam cerca de $90 \%$ do total de recursos negociados o que pode reforçar a desigualdade regional. Visto que a capacitação científica e tecnológica historicamente está concentrada nas regiões Sudeste e Sul, essas atraem para si maior número de professores doutores, recursos e infraestrutura para pesquisa, e esse processo cumulativo retroalimenta o sistema, levando ao estabelecimento de mecanismos de auto-reforço da concentração.

Tabela 4 - Distribuição dos contratos de transferência de tecnologia por objeto, montante e distribuição

\begin{tabular}{|c|c|c|c|c|c|c|c|c|c|c|c|}
\hline \multirow[t]{2}{*}{ Objeto } & \multicolumn{2}{|r|}{ Sul } & \multicolumn{2}{|c|}{ Sudeste } & \multicolumn{2}{|c|}{ Centro-oeste } & \multicolumn{2}{|c|}{ Nordeste } & \multicolumn{2}{|r|}{ Norte } & \multirow{2}{*}{$\begin{array}{c}\begin{array}{c}\text { Total } \\
\text { (por objeto) }\end{array} \\
\text { Valor (R\$) } \\
(1.000 .000)\end{array}$} \\
\hline & Qtde. & $\begin{array}{c}\text { Valor (R\$) } \\
(1.000 .000)\end{array}$ & Qtde. & $\begin{array}{c}\text { Valor (R\$) } \\
(1.000 .000) \\
\end{array}$ & Qtde. & $\begin{array}{c}\text { Valor (R\$) } \\
(1.000 .000)\end{array}$ & Qtde. & $\begin{array}{c}\text { Valor (R\$) } \\
(1.000 .000)\end{array}$ & Qtde. & $\begin{array}{c}\text { Valor (R\$) } \\
(1.000 .000)\end{array}$ & \\
\hline $\begin{array}{l}\text { Contrato de licenciamento de direitos de } \\
\text { propriedade intelectual (patente; desenho } \\
\text { industrial; marca; programa de computador; } \\
\text { topografia de circuito integrado; cultivar; obra } \\
\text { literária, artística ou científica; outros). }\end{array}$ & 91 & 1,64 & 59 & 21,52 & 669 & 10,90 & 3 & 0,35 & 1 & 0,00 & 34,42 \\
\hline $\begin{array}{l}\text { Acordo de parceria de pesquisa, } \\
\text { desenvolvimento e inovação (atividades } \\
\text { conjuntas de pesquisa científica, e/ou } \\
\text { tecnológica e desenvolvimento de tecnologia, } \\
\text { produto ou processo). }\end{array}$ & 365 & 119,63 & 104 & 95,77 & 10 & 6,04 & 6 & 0,30 & 0 & 0,00 & 221,74 \\
\hline $\begin{array}{l}\text { Contrato de know-how (envolvendo ativos } \\
\text { intangíveis não amparados por direito de PI), } \\
\text { assistência técnica (contratação de soluções } \\
\text { técnicas ou capacitação ou treinamento) e } \\
\text { demais serviços. }\end{array}$ & 76 & 36,58 & 81 & 71,46 & 1 & 0,00 & 1 & 0,00 & 0 & 0,00 & 108,04 \\
\hline $\begin{array}{l}\text { Contrato de cessão de direitos de propriedade } \\
\text { intelectual (patente; desenho industrial; } \\
\text { marca; programa de computador; topografia } \\
\text { de circuito integrado; cultivar; obra literária, } \\
\text { artística ou científica; outros). }\end{array}$ & 2 & 0,00 & 0 & 0,00 & 0 & 0,00 & 0 & 0,00 & 0 & 0,00 & 0,00 \\
\hline $\begin{array}{l}\text { Contrato ou convênio de uso do capital } \\
\text { intelectual em projetos de pesquisa, } \\
\text { desenvolvimento e inovação. }\end{array}$ & 27 & 21,84 & 2 & 0,34 & 16 & 0,36 & 0 & 0,00 & 0 & 0,00 & 22,20 \\
\hline TOTAL & 561 & 179,69 & 246 & 189,09 & 696 & 52,78 & 10 & 0,65 & 1 & 0,00 & 422,21 \\
\hline
\end{tabular}

Fonte: Elaborada pelos autores a partir do FORMICT, 2017.

Essa conjectura faz ainda mais sentido após a verificação de que o percentual de grupos de pesquisa que declararam ter envolvimento com empresas em relação ao número de grupos total cadastrado no diretório de grupos do CNPq é semelhante para as cinco regiões do Brasil, mas que sua ocorrência está concentrada nas regiões Sudeste e Sul, conforme Tabela 5. 
Tabela 5 - Distribuição do no de grupos de pesquisa que relataram pelo menos um relacionamento com empresa, segundo região geográfica onde o grupo está localizado

\begin{tabular}{|l|c|c|c|c|c|c|}
\hline \multirow{2}{*}{ Região } & \multicolumn{2}{|c|}{$\begin{array}{c}\text { Total de grupos na } \\
\text { região (a) }\end{array}$} & \multicolumn{2}{c|}{$\begin{array}{c}\text { No grupos que relataram } \\
\text { relacionamento (b) }\end{array}$} & \multicolumn{2}{c|}{ b/a*100 } \\
\hline & 2004 & 2014 & 2004 & 2014 & 2004 & 2014 \\
\hline Centro-Oeste & 1.139 & 2.654 & 134 & 739 & 11,76 & 27,80 \\
\hline Sudeste & 10.221 & 15.549 & 965 & 4.203 & 9,44 & 27,00 \\
\hline Sul & 4.580 & 7.938 & 611 & 2.111 & 13,34 & 26,60 \\
\hline Nordeste & 2.760 & 7.215 & 352 & 1.792 & 12,75 & 24,80 \\
\hline Norte & 770 & 2.068 & 89 & 503 & 11,56 & 24,30 \\
\hline Total & 19.470 & 35.424 & 2.151 & 9.348 & - & - \\
\hline
\end{tabular}

Fonte: Elaborada pelos autores a partir do DG CNPq, 2017.

Desse modo, as regiões Sudeste e Sul teriam cooperações mais profícuas com empresas, seja por sua capacitação científica, produção de tecnologias e/ou competência para negociá-las. Pode contribuir para esse processo cumulativo ${ }^{9}$ (Fagundes, Cavalcante \& Ramjaccioti, 2005) a entrada de maior fluxo de recursos a partir dos rendimentos dos contratos de tecnologia (que possivelmente já se configura como resultado da dinâmica descrita acima).

Com relação aos rendimentos relativos à transferência de tecnologia no ano de $2016^{10}$, as ICTS públicas informaram ter recebido aproximadamente $R \$ 102$ milhões e as instituições privadas contabilizaram R\$ 125 milhões. Entretanto, a partir dos dados disponibilizados no FORMICT, não é possível identificar quais contratos são responsáveis por esse rendimento. Já os gastos que as instituições tiveram em 2016 com registros e manutenção de proteções de propriedade intelectual, foram, respectivamente, de $R \$ 8$ e de $R \$ 1,4$ milhão.

A partir da comparação entre rendimentos e gastos das instituições públicas e privadas, é possível supor que estas parecem ser mais eficientes que as primeiras uma vez que o seu gasto com os DPI representa 1,12\% de seu rendimento, enquanto que, nas instituições públicas, esse valor é de aproximadamente 7,84\%. Esta comparação sugere que as instituições públicas podem, tipicamente, estar registrando e mantendo tecnologias que não estão sendo transferidas e/ou gerando resultados. Essa prática já havia sido sugerida por Dalmarco et al. (2011), que acreditam que muitas patentes protegidas pelas universidades, por não terem uma avaliação adequada de mercado, acabam sendo arquivadas.

No tocante aos indicadores de resultados da transferência de tecnologia, dados da PINTEC (2016) revelam que a taxa de inovação empreendida pelas empresas brasileiras foi de $36 \%$ no período de 2012 a 2014, não registrando alterações em relação ao triênio imediatamente anterior, no qual a taxa foi de $35,7 \%$. No que diz respeito às atividades inovativas realizadas pelas empresas para viabilização de suas inovações, observa-se que a taxa relativa às atividades internas de P\&D ainda são relativamente baixas, o que dificulta absorção de tecnologia no caso de interações com ICTs. Além

\footnotetext{
${ }^{9}$ Esse processo foi abordado também por Lima, Muniz, Oliveira \& Cruz (2012) ao discutirem as desigualdades científicas regionais entre as universidades públicas da Bahia.

${ }^{10}$ Esses valores consideram os rendimentos referentes aos contratos firmados no ano-base de 2016 e que geraram recursos em 2016 ; ou que tenham sido firmados em anos anteriores, mas geraram recursos no ano de 2016 (FORMICT, 2017).
} 
disso, o tipo de aquisição que tem maior importância relativa para as empresas que implementaram inovação em produto e processo são de máquinas e equipamentos com $72,5 \%$, o que exclui a participação de transferência de tecnologias por ICTs.

Esse quadro demonstra que o crescimento nos indicadores de esforços para o desenvolvimento tecnológico ainda não tem refletido em resultados efetivos e reforça a importância do estabelecimento de interação entre ICTs e empresas, não apenas em número, mas na qualidade da parceria, e, principalmente, numa perspectiva sistêmica de inovação. Pois, seguindo a abordagem linear de inovação, o conhecimento técnico produzido no ambiente acadêmico pode até ser protegido, mas é mais difícil de ser transferido para o setor produtivo, situação que parece explicar os dados do PINTEC e o alto percentual de gastos com DPI das instituições públicas em relação aos rendimentos com as transferências tecnológicas, apresentados anteriormente.

\section{Discussão dos resultados}

A partir das informações prestadas pelas ICTs ao FORMICT, foi possível identificar que as ICTs públicas têm maior número de contratos de tecnologia que as instituições privadas, o que confirma a relevância daquelas instituições para geração de novos conhecimentos, conforme pontuado Etzkowitz \& Zhou (2017) e Perkmann et al. (2013).

O número de contratos de transferência de tecnologia é expressivo em relação ao total de contratos declarados pelas ICTs, o que se apresenta em linha com o maior dinamismo nas atividades de patenteamento e comercialização tecnológica das ICTs constatado por Viana et al. (2018). Daqueles, os contratos de licenciamento de direito de propriedade intelectual foram os que se apresentaram em maior quantidade, seguidos dos contratos de know how e cessão de DPI, o que sugere que o aumento do número de relacionamentos com empresas (pelo menos por parte das universidades) e da proteção patentária registrado no Diretório de Grupos do CNPq e site do INPI, respectivamente, está gerando resultados.

Esse cenário revela avanços na estruturação de atividades que viabilizam a governança de transações tecnológicas pelas ICTs, conforme preconizado por UNCTAD (2014) e Zucoloto (2013). Entretanto, dados divulgados pela PINTEC em 2016 revelaram que praticamente não houve alteração da taxa de inovação empreendida pelas empresas brasileiras nos últimos dois triênios, ou seja, o progresso tecnológico das empresas nacionais ainda se mostra, de forma geral, tímido.

De maneira mais ampla, Viana et al. (2018) atribuem esse quadro a ausência de legislação clara e específica de estímulo à TT, baixa autonomia, infraestrutura adequada e pessoal com capacidade técnica nos NITs para estudos de viabilidade econômica e técnica das tecnologias desenvolvidas. Sousa et al, (2018), por sua vez, pontuam o distanciamento das pesquisas realizadas no âmbito acadêmico da aplicação comercial demandada pelo mercado e falta de postura empreendedora e limitada utilização das estratégias de comunicação das universidades. Ainda que 
todas essas barreiras sejam ultrapassadas, a empresa necessita ter conhecimento para utilizar a tecnologia adquirida, ou seja, a capacidade de absorção explicitada em UNCTAD (2014) e Amesse and Cohendet (2001). Ademais, o fortalecimento da cultura de PI e TT e o aprendizado de seus mecanismos é um processo que exige mudança de postura das instituições envolvidas (ICTs e setor produtivo), não ocorrendo em curto espaço de tempo.

Os contratos de acordo de parceria, desenvolvimento e inovação apresentam maior montante de recursos negociados. Isso pode estar relacionado ao fato de o pagamento deste tipo de contratação ser feito no início do processo de pesquisa, enquanto que o de licenciamento e cessão de DPI geralmente é realizado após a disponibilização da tecnologia no mercado. Assim, não nos parece razoável considerar que os contratos que apresentaram maior volume de recursos no período sejam os de maiores resultados.

Ainda, com relação ao montante de recursos negociados, foi possível perceber que seus maiores índices estão concentrados nas instituições localizadas no Sul e Sudeste do país. Isso pode estar relacionado a maior background de instituições situadas nessas regiões em relação à temática. Afinal, o incremento nas atividades de patenteamento e comercialização tecnológica das ICTs paulistas já havia sido constatada em estudo por Garnica e Torkomian (2009).

Dado que as cinco regiões têm semelhante índice de envolvimento dos grupos de pesquisa com empresas em relação ao número total cadastrado no diretório de grupos do CNPq, é importante salientar que os contratos de tecnologia podem ser mais um fator de reforço das desigualdades entre as regiões, uma vez que as do Sul e do Sudeste são as que tradicionalmente têm maior número de programas de pós-graduação bem avaliados, doutores, grupos de pesquisa, cooperações mais profícuas e, portanto, maior fluxo de recursos

A proporção entre as despesas com DPI e rendimentos relativos à transferência tecnológica é aproximadamente três vezes menor nas instituições privadas do que nas públicas, o que sugere que estas instituições podem estar registrando e mantendo tecnologias que não estão sendo transferidas e/ou gerando resultados, conforme pontuado Dalmarco et al. (2011). O que sugere que as ICTs continuam mantendo um comportamento ainda consonante com o modelo linear de inovação.

\section{Considerações finais}

O presente trabalho retrata os contratos de TT realizados por ICTs no Brasil a partir das informações prestadas por estas no Relatório FORMICT de 2017 e explora a sua relação com outras variáveis pertinentes à TT a partir dos dados disponibilizados pelo Diretório de Grupos de Pesquisa do CNPq, INPI e PINTEC

De modo geral, os resultados da pesquisa contribuem para a compreensão de que as políticas de incentivo à CT\&I no Brasil têm contribuído para um amadurecimento das ICTs no que se refere à proteção de sua propriedade intelectual, mas ainda não foi capaz de promover a inovação tecnológica 
de forma substantiva. Apesar de os dados relativos aos contratos de tecnologias do FORMICT indicarem um movimento mais contundente para a transposição do conhecimento de base científico para a produção, do ponto de vista prático, a efetividade desse processo exige o estabelecimento de vínculos mais intensos e extensos de interação entre ICTs e empresas numa perspectiva sistêmica de inovação. Além disso, é necessário um relativo período de tempo para que as tecnologias transferidas das ICTs para empresas superem os desafios mercadológicos e sejam efetivamente implementadas.

Cumpre destacar, que a não padronização das informações divulgadas e, principalmente, o fato dessas não se constituírem resultados de uma mesma amostra de instituições respondentes do FORMICT não possibilitou a análise da evolução dos contratos das ICTs ao longo do tempo, o que representa uma limitação do estudo. Além da ampliação do período de estudo, sugere-se que novos trabalhos explorarem os fatores que contribuem para o descompasso entre a capacidade científica, aplicação tecnológica e desenvolvimento de inovação pelas empresas e, finalmente, quais políticas poderiam ser adotadas para catchup das regiões Norte, Nordeste e Centro-Oeste no que diz respeito a esses indicadores.

\section{Referências}

AMESSE, F. \& COHENDET, P. (2001). Techonoly transfer revisited from the perspective of the knowledge-based economy. Research Policy. V.30. 1459-1478. Disponível em: https://doi.org/10.1016/S0048-7333(01)00162-7. Acesso em 20 jan. 2016.

BARBOSA, D. B. (2003). Uma introdução à propriedade intelectual. 2. ed. Rio de Janeiro: Lumen Juris.

CALDERAN, L. L. \& GUILHERME, L.O. (2013). A inovação e a interação Universidade-Empresa: uma revisão teórica. Série Textos para a discussão CEAG/UnB. Centro de Estudos avançados de Governo e de Administração Pública-CEAG. Brasília.

CARVALHO, N. (2014). A gestão da cooperação na integração entre universidade-empresa-governo: fatores facilitadores da tríplice hélice. Anais do Simpósio de Gestão da Inovação Tecnológica. Belo Horizonte. MG, Brasil, 28.

CHESBROUGH, H. W. (2003). Open innovation: the new imperative for creating and profiting from technology. Boston, MA: Havard Business School Press.

CLOSS, L. FERREIRA, G; SAMPAIO \& C. PERIN, M. (2012). Intervenientes na Transferência de Tecnologia Universidade-Empresa: o Caso PUCRS., v. 16, n. 1, art. 4, pp. 59-78, Jan./Fev.

CUNICO, E.; CIRANI, C. B. S. \& TEIXEIRA, C. E. (2015). A Relação Universidade-Empresa: os entraves nas inter-relações entre agentes do SNI em um projeto de cooperação. Anais do Encontro da Associação Nacional de Pós-Graduação e Pesquisa em Administração. Belo Horizonte. MG, Brasil, 39.

DALMARCO, G., DEWES, M. F., ZAWISLAK, P. A., \& PADULA, A. D. (2011). Universities' intellectual property: path for innovation or patent competition? Journal of Technology Management \& Innovation, 6(3), 159-170. Disponível em: http://dx.doi.org/10.4067/S0718-27242011000300012. Acesso em 10 dez. 2016. 
DIAS, A. A., \& PORTO, G. S. (2014). Como a USP transfere tecnologia? Organizações \& Sociedade, 21(70), 489-508.

ETZKOWITZ, H. \& ZHOU, C. (2017). Hélice Tríplice: inovação e empreendedorismo universidadeindústria-governo. Estudos Avançados, v. 31, n. 90, p. 23-48. Disponível em: https://doi.org/10.1590/s0103-40142017.3190003. Acesso em 10 jan. 2019.

ETZKOWITZ, H, H; LEYDESDORFF, L. (2000). The dynamics of innovation: from national systems and "mode2" to a triple helix of university-industry-government relations. Research Policy, v. 29, p. 109-123. Disponível em: https://doi.org/10.1016/S0048-7333(99)00055-4. Acesso em 10 set. 2013.

FAGUNDES, M. E. M.; CAVALCANTE, L. R.; RAMJACCIOTI, R. E. L. (2005). Desigualdades regionais em ciência e tecnologia no Brasil. Bahia Análise \& Dados, v. 14, n. 4, p. 755-768.

FREEMAN, C. (1998). Innovation systems: city-state, national, continental and sub-national. Nota Técnica - Instituto de Economia da Universidade Federal do Rio de Janeiro, Rio de Janeiro.

FREEMAN „, C. (1995). The "National System of Innovation" in historical perspective. Cambridge Journal of Economics, v.19, p. 5-24.

GARNICA, L. A., \& TORKOMIAN, A. L. V. (2009). Gestão de tecnologia em universidades: Uma análise do patenteamento e dos fatores de dificuldades e de apoio à transferência de tecnologia no Estado de São Paulo. Gestão \& Produção, 16(4), 624-638.

GIL, A. C. (2002). Como elaborar projetos de pesquisa. 6.e. São Paulo: Atlas. 175p.

GOMES, M. S. GONÇALO, C. R.; PEREIRA, C. D. \& VARGAS, S. L. (2014). A inovação como conexão para o desenvolvimento de parcerias entre universidade-empresa. (2014). Navus - Revista de Gestão e Tecnologia. Florianópolis, SC, v. 4, n. 2, p. 78-91.

IBGE. (2016). Pesquisa de Inovação. Pintec. Rio de Janeiro: IBGE - Instituto Brasileiro de Geografia e Estatística.

INSTITUTO NACIONAL DA PROPRIEDADE INDUSTRIAL (Brasil). Estatísticas. Estatísticas preliminares - a partir de 2013. Disponível em: < http://www.inpi.gov.br/estatisticas/estatisticas-preliminares-2013a-partir-de-2013>. Acesso em 06 jun. 2018.

INSTITUTO NACIONAL DA PROPRIEDADE INDUSTRIAL (Brasil). Estatísticas. Estatístico de Propriedade Industrial 2000-2012. Disponível em: < http://www.inpi.gov.br/estatisticas/anuario-estatistico-depropriedade-industrial-2000-2012>. Acesso em 06 jun. 2018.

Lei n. 10.973 de 02 de dezembro de 2004. Dispõe sobre Inovação Tecnológica. Congresso Nacional. Atos do Poder Legislativo, DOU, n.․ 232 de 03.12.2004. Disponível em: <http://www.planalto.gov.br/ccivil 03/leis/L9279.htm. Acesso em 03 de dezembro de 2015.

Lei n.o 9.279 de 14 de maio de 1996. Dispõe sobre Propriedade Intelectual. Congresso Nacional. Disponível em: <http://www.planalto.gov.br/ccivil 03/leis/L9279.htm. Acesso em 03 de dezembro de 2015.

LEMOS, D. C. \& CARIO, S. A. F. (2015). Os Sistemas Nacional e Regional de Inovação e sua influência na Interação Universidade-Empresa em Santa Catarina. Anais do Encontro da Associação Nacional de Pós-Graduação e Pesquisa em Administração, Belo Horizonte, NG, Brasil, 39. 
LIMA, P. R. L.; MUNIZ, M. M. de J.; OLIVEIRA, E. S. M. C. O; CRUZ, R. (2012). Desigualdades Científicas Regionais Entre as Universidades Públicas da Bahia. Anais do Encontro Acadêmico de Propriedade Intelectual, Inovação e Desenvolvimento, Rio de Janeiro, RJ, Brasil, 5.

LUNDVALL, B. (2005). National innovation systems - analytical concept and development tool. Proceedings of the Druid Summer Conference. Copenhagen. Disponivel em: <http://www.druid.dk/conferences/Summer2005/Papers/Lundvall.pdf>. Acesso em 10 ago. 2014.

LUZ, M. C. V. da. (2015). Contratos de tecnologia: perspectiva de análise e questões de pesquisa. Anais do Seminário Internacional de Inovação na Pequena e Média Empresa. João Amato Neto (org.). São Paulo, SP, Brasil, 3. Disponível em: http://pro.poli.usp.br/wp-content/uploads/2015/10/Livro-IIISIIPME.pdf. Acesso em 26 de outubro de 2015.

MCTI. (2017). Política de Propriedade Intelectual das Instituições Científicas e tecnológicas do Brasil. Relatório Formict 2016. Brasília. Disponível em: http: https://www.mctic.gov.br/mctic/export/sites/institucional/tecnologia/propriedade_intelectual/arq uivos/Relatorio-Formict-Ano-Base-2016.pdf. Acesso em 20 de outubro de 2018.

MOWERY, D. C. \& SAMPAT, B. N. Universities in national innovation systems. In: FAGERBERG, J; MOWERY, D. C; NELSON, R. R. (2010) The Oxford handbook of innovation. Oxford: Oxford. Disponível em: $<$ http://innovate.ucsb.edu/wp-content/uploads/2010/04/Mowery-Sampat-UnivNational-Innovation-Systems.pdf >. Acesso em 12 outubro 2013.

NELSON, R. R \& NELSON, K. (2002). Technology, institutions, and innovation systems. Research Policy, V. 31, p. 265-272.

NOVELI, M. \& SEGATTO, A. P. (2012). Processo de Cooperação Universidade Empresa para a Inovação Tecnológica em um Parque Tecnológico: Evidências Empíricas e Proposição de um Modelo Conceitual. Revista de Administração e Inovação. v. 1 n. 1. p. 81-105.

PERKMANN, M. et al. (2013). Academic engagement and commercialization: A review of the literature on university-industry relations. Research Policy, v. 42, n. 2, p. 423-442.

PERKMANN, M., NEELY, A. \& WALSH, K. (2011). How should firms evaluate success in university industry alliances? A performance measurement system. R\&D Management, v. 41, p. 202-216. Disponível em: https://doi.org/10.1016/j.respol.2012.09.007. Acesso em 10 set. 2013.

PUFFAL, D. P; TREZ, J. R \& SCHAEFFER, P. R. (2012). Características da interação universidade-empresa no Brasil: motivações e resultados sob a ótica dos envolvidos. Anais do Simpósio de Gestão da Inovação Tecnológica da ANPAD, Salvador, BA, Brasil, 7.

SCHUMPETER, J. A. (1961). Capitalismo, socialismo e democracia. Rio de Janeiro: Fundo de Cultura. $488 p$.

SORIA, A. F. \& FERREIRA, G. C. (2012). Formas de Transferência de Tecnologia na Interação Universidade-Empresa: O Caso PUCRS. Anais do Simpósio de Gestão da Inovação Tecnológica, Salvador, BA, Brasil, 27.

SOUSA, D.; ZAMBALDE, A.; SOUKI, G. \& VERONEZE, R. (2018). Marketing Myopia in Brazilian Public Universities: An Empirical Study Involving Academicians. Journal of Technology Management \& Innovation, v. 13, n. 3, p. 12-23. Disponível em: http://dx.doi.org/10.4067/s071827242018000300012. Acesso em 20 jan. 2019. 
UNCTAD. (2014). Transfer of Technology and Knowledge Sharing for Development Science: technology and innovation issues for developing countries. n. 8.

VIANA, L. et al. (2018). Patents Go to The Market? University-Industry Technology Transfer from a Brazilian Perspective. Journal of technology management \& innovation, v. 13, n. 3, p. 24-35. Disponível em: https://doi.org/10.4067/S0718-27242018000300024. Acesso em 20 jan. 2019.

ZUCOLLOTO, G. F. (2013). Propriedade Intelectual em debate. Radar: tecnologia, produção e comércio exterior, $\quad$ n. 29, out. Disponível em: http://ipea.gov.br/portal/images/stories/PDFs/radar/131009_radar29_cap1 >. Acesso em 16 de dezembro 2015.

Recebido em: 14 mar. 2019 / Aprovado em: 09 out. 2019

Para referenciar este texto

American Psychological Association (APA)

Almeida, M. M. de J., Luz, M. C. V. da, \& Quintella, R. H. (2020, out./dez.). Relação UniversidadeEmpresa: perfil dos contratos de tecnologia e sua distribuição regional segundo dados do FORMICT. Exacta, 18(4), 799-819. https://doi.org/10.5585/exactaep.v18n4.13348. 